\title{
EXPERIMENTAL INVESTIGATION ON THE MECHANICAL PROPERTIES OF CONCRETE MIXED WITH BANANA STEM FIBER AS WELL AS HYBRID STEEL FIBER
}

\author{
S. Prakash Chandar*, K. Gunasekaran, V.P. Nabeel Babu \\ and Ramesh Potti \\ Department of Civil Engineering, SRM IST, Chennai \\ *E-mail: nabeelfrk@gmail.com
}

\begin{abstract}
Fibers have been used to reinforce concrete over the last few decades because fiber has the significant influence on concrete static and dynamic properties. There are several fiber reinforcement methods for improving the performance of concrete, which lacks in load carrying stability and soundness. Use of banana stem fiber and steel fiber will have greater improvement in the properties of concrete. So the comparison of banana stem fiber and steel fiber reinforced concrete (SFRC) was the basic concern of this study and also how these fibers influence concrete properties by inclusion of banana stem fibers and steel fibers in percentage 0.5 to 2.5. For this purpose cubes, cylinder and flexural beams were cast and carried out the test under the different testing machine for compressive, tensile, flexural, impact, and bond strength. The result obtained from the experiments had been analyzed and compared with the conventional concrete (CC).
\end{abstract}

Keywords: Banana stem fiber, steel fiber, reinforcement, impact strength, renewable resource

(c) RASĀYAN. All rights reserved

\section{INTRODUCTION}

At present concrete is one of the broadly used materials in the field of construction industry. The reason for this because of its availability can be manufactured in any desired shape, low maintenance, affordability, and also it has better compressive strength. As we know concrete is stronger in compressive strength in comparison to the tensile strength. So this may create some cracks in concrete while carrying the load. Due to this many studies have been done to utilize fiber as reinforcement materials in concrete by altering the mix proportion and the inclusion of foreign materials will help the concrete to gain specific strength and soundness. In which, fiber reinforcement is considered as promising and superior to any other constituents in modifying basic properties of concrete due to its availability and low consumption of energy. Fiber reinforced concrete can settle the constraints of the normal concrete e.g. lack of tensile strength, less toughness, less post cracking capacity. The foremost role of fiber in concrete is to bridge the crack, reduce shrinkage cracking. So the fiber reinforcement will help to create a concrete, which has superior characteristics and properties to that of normal concrete. So the application of fiber reinforced concrete is getting higher in various fields.

\section{EXPERIMENTAL}

\section{Material used}

Cement (53opc), river sand, coarse aggregate, banana stem fibers and steel fibers. The fine and coarse aggregate materials used in this project are locally available.

\section{Cement}

Ordinary Portland cement of 53 grades was the major constituents of all the trail mixes (with or without fibers). This grade cement bought from the store.

\section{Sand}

The sand used in concrete is conforming to zone III of IS: 383:1997. Then the sand was sieved through $4.75 \mathrm{~mm}$. The specific gravity of sand was 2.63 . 


\section{Coarse Aggregate}

The coarse aggregate was used $20 \mathrm{~mm}$. Crushed granite gravel obtained from the local market was used as coarse aggregate. The specific gravity of coarse aggregate was 2.74 .

\section{Banana Stem Fiber}

Banana stem fiber of diameter $1 \mathrm{~mm}$ and length of $40 \mathrm{~mm}$ was used. The aspect ratio of banana stem Fiber was 40 . The specific gravity of banana stem fiber was 1.35 .

\section{Steel Fiber}

The fiber is used as hooked end steel fibers. Steel fibers used are of aspect ratio 50 with diameter $1 \mathrm{~mm}$ and length $50 \mathrm{~mm}$. The specific gravity of steel fiber was 7.85 .

\section{RESULTS AND DISCUSSION}

Concrete mix of 1:1:2 was used with 0.45 water-cement ratio. For each mix, the test specimen was prepared for testing at 3,7 and 28 days. These experiments were conducted using a different amount of fibers.

\section{Compressive Strength Test}

The size of the cube specimen was $150 \mathrm{~mm} \times 150 \mathrm{mmx} 150 \mathrm{~mm}$. The test specimens were placed in the CTM and the load applied was truly axial.

Table-1: Compressive strength of CC, Banana stem fiber concrete and SRFC

\begin{tabular}{|c|c|c|c|c|}
\hline \multicolumn{5}{|c|}{ Compressive strength $\left(\mathrm{N} / \mathrm{mm}^{2}\right)$} \\
\hline Type of Mix & Fiber $(\%)$ & $3^{\text {rd }}$ day & $7^{\text {th }}$ day & $28^{\text {th }}$ day \\
\hline Normal Concrete & 0 & 13.25 & 19.78 & 29.91 \\
\hline \multirow{5}{*}{$\begin{array}{l}\text { Concrete with inclusion of } \\
\text { Banana stem fiber }\end{array}$} & 0.5 & 14.83 & 22.51 & 31.63 \\
\hline & 1 & 15.91 & 26.75 & 35.55 \\
\hline & 1.5 & 14.56 & 21.63 & 29.45 \\
\hline & 2 & 13.10 & 19.39 & 22.76 \\
\hline & 2.5 & 11.63 & 15.81 & 18.91 \\
\hline \multirow{5}{*}{$\begin{array}{c}\text { Concrete with inclusion of Steel } \\
\text { fiber }\end{array}$} & 0.5 & 18.71 & 23.63 & 32.37 \\
\hline & 1 & 24.11 & 34.54 & 38.73 \\
\hline & 1.5 & 17.01 & 28.31 & 35.33 \\
\hline & 2 & 16.31 & 21.80 & 28.72 \\
\hline & 2.5 & 16.0 & 20.3 & 25.16 \\
\hline
\end{tabular}

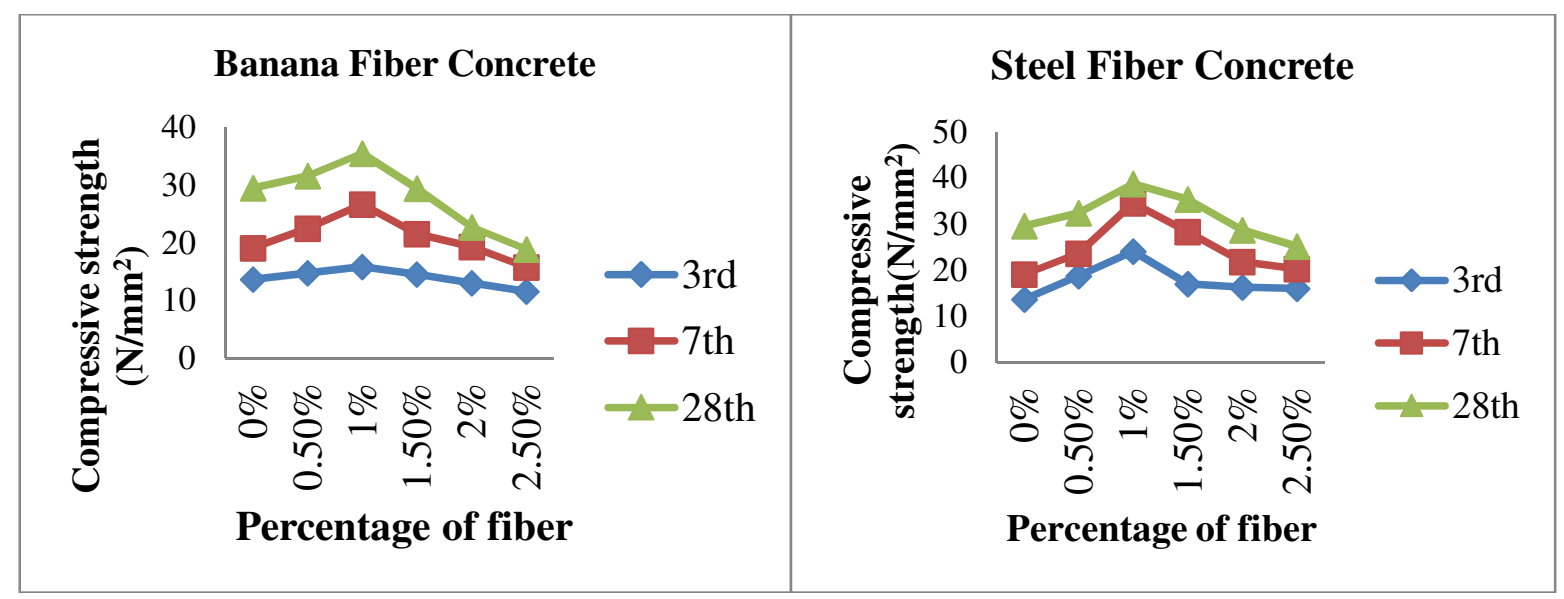

Fig.-1: Line diagram for compressive strength of banana stem fiber and SFRC 


\section{Split Tensile Strength Test}

For each mix, a cylinder with $300 \mathrm{~mm}$ height and $150 \mathrm{~mm}$ diameter was prepared for testing at 3,7 and 28 days. The ultimate load has been recorded and determines the split tensile strength of concrete.

Table -2: the Split tensile strength of CC, Banana stem fiber concrete and SRFC

\begin{tabular}{|c|c|c|c|c|}
\hline \multicolumn{5}{|c|}{ Split tensile strength $\left(\mathrm{N} / \mathrm{mm}^{2}\right)$} \\
\hline Type of Mix & Fiber $(\%)$ & $3^{\text {rd }}$ day & $7^{\text {th }}$ day & $28^{\text {th }}$ day \\
\hline Normal Concrete & 0 & 1.10 & 1.83 & 3.01 \\
\hline \multirow{5}{*}{$\begin{array}{l}\text { Concrete with inclusion of Banana } \\
\text { stem fiber }\end{array}$} & 0.5 & 1.40 & 2.81 & 3.01 \\
\hline & 1 & 1.46 & 2.95 & 3.96 \\
\hline & 1.5 & 1.32 & 2.65 & 3.72 \\
\hline & 2 & 1.18 & 2.32 & 3.60 \\
\hline & 2.5 & 1.16 & 2.16 & 3.43 \\
\hline \multirow{5}{*}{$\begin{array}{l}\text { Concrete with inclusion of Steel } \\
\text { fiber }\end{array}$} & 0.5 & 1.52 & 2.84 & 3.83 \\
\hline & 1 & 1.68 & 2.97 & 3.98 \\
\hline & 1.5 & 1.43 & 2.69 & 3.69 \\
\hline & 2 & 1.38 & 2.36 & 3.68 \\
\hline & 2.5 & 1.25 & 2.24 & 3.54 \\
\hline
\end{tabular}

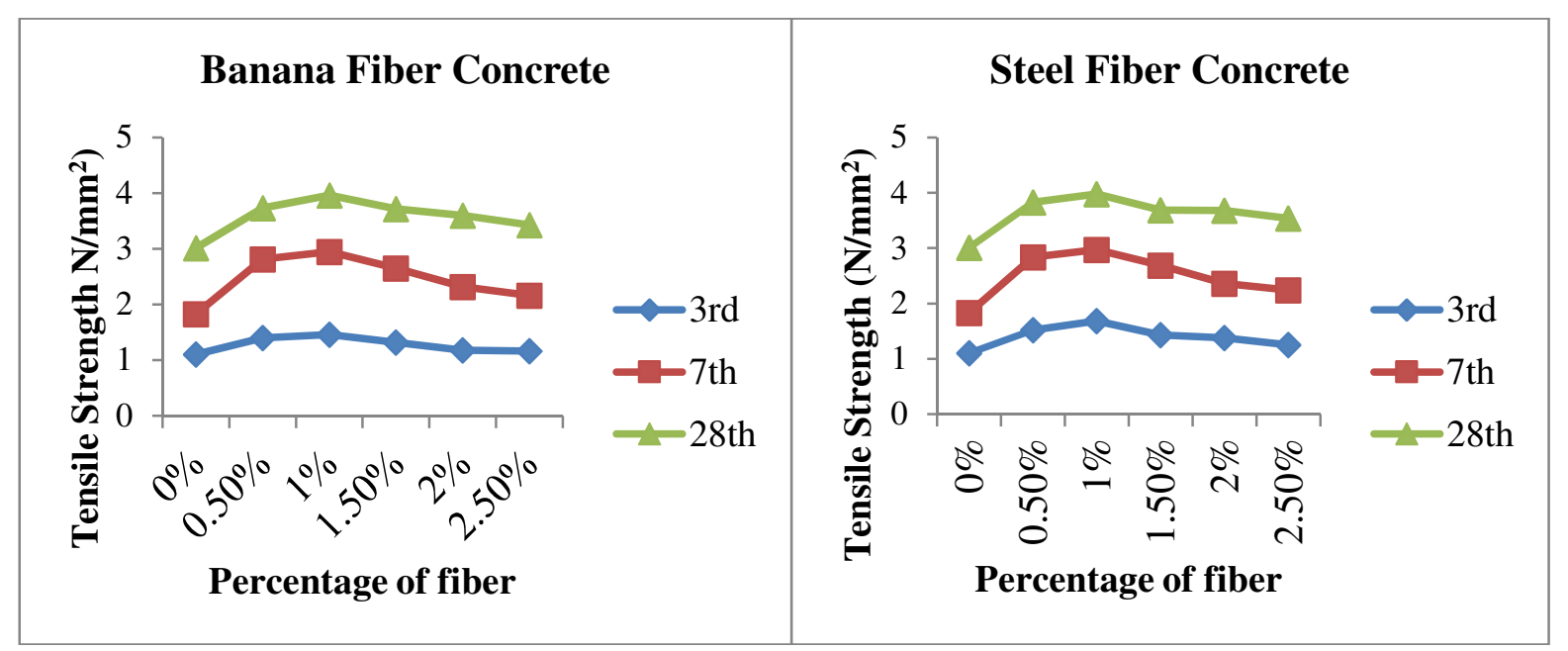

Fig.-2: Line diagram for the split tensile strength of banana stem fiber and SFRC concrete.

\section{Flexural Strength Test}

For this test, the beam size of $100 \mathrm{mmx} 100 \mathrm{mmx} 500 \mathrm{~mm}$ was used for testing at 3, 7 and 28 days. During this test, maximum applied load was recorded to calculate the flexural strength.

Table-3: Flexural strength of CC, Banana stem fiber concrete and SRFC

\begin{tabular}{c|c|c|c|c}
\hline \multicolumn{5}{c}{ Flexural strength $\left(\mathrm{N} / \mathrm{mm}^{2}\right)$} \\
\hline \multirow{2}{*}{ Type of Mix } & Fiber $(\%)$ & $3^{\text {rd }}$ day & $7^{\text {th }}$ day & $28^{\text {th }}$ day \\
\hline Normal Concrete & 0 & 2.1 & 3.1 & 5 \\
\hline \multirow{3}{*}{$\begin{array}{c}\text { Concrete with inclusion of Banana } \\
\text { stem fiber }\end{array}$} & 0.5 & 2.2 & 3.19 & 6.1 \\
\cline { 2 - 5 } & 1 & 2.8 & 3.93 & 6.8 \\
\cline { 2 - 5 } & 1.5 & 2.5 & 3.74 & 6.4 \\
\cline { 2 - 5 } & 2 & 2.4 & 3.38 & 6.3 \\
\hline
\end{tabular}


RASĀYAN J. Chem.

Vol. 11 | No. 2 |640 - 646 | April - June | 2018

\begin{tabular}{c|c|c|c|c}
\hline & 2.5 & 2.2 & 3.20 & 6.1 \\
\hline \multirow{3}{*}{$\begin{array}{c}\text { Concrete with inclusion of Steel } \\
\text { fiber }\end{array}$} & 0.5 & 2.7 & 4 & 7.3 \\
\cline { 2 - 5 } & 1 & 2.9 & 4.4 & 7.5 \\
\cline { 2 - 5 } & 1.5 & 2.83 & 4.3 & 7 \\
\cline { 2 - 5 } & 2 & 2.76 & 4.2 & 6.6 \\
\cline { 2 - 5 } & 2.5 & 2.73 & 4.2 & 6.3 \\
\hline
\end{tabular}

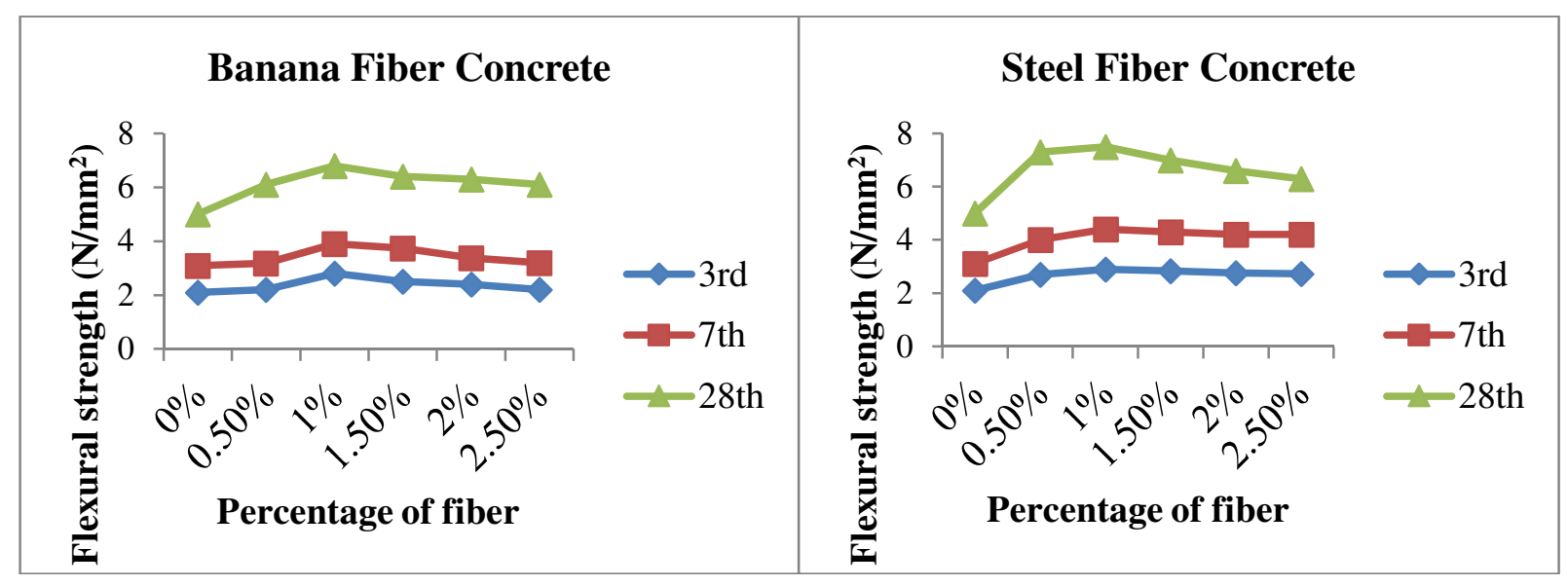

Fig.-3: Line diagram for flexural strength of banana stem fiber and SFRC concrete

\section{Impact Strength Test}

For this test, a disc with $63.5 \mathrm{~mm}$ thick and $152.4 \mathrm{~mm}$ in diameter was used. This test was performed in accordance with the guidelines of ACI committee 544 specifications.

Table-4: Impact strength of CC, Banana stem fiber concrete and SRFC

\begin{tabular}{|c|c|c|c|c|c|c|c|}
\hline \multicolumn{8}{|c|}{ Impact Strength } \\
\hline \multirow[t]{2}{*}{ Type of Mix } & \multirow[t]{2}{*}{ Fiber $(\%)$} & \multicolumn{2}{|c|}{$\begin{array}{c}3^{\text {rd day }} \\
\text { (No. of blows) }\end{array}$} & \multicolumn{2}{|c|}{$\begin{array}{c}7^{\text {th }} \text { day } \\
\text { (No. of blows) }\end{array}$} & \multicolumn{2}{|c|}{$\begin{array}{c}28^{\text {th }} \text { day } \\
\text { (No. of blows) }\end{array}$} \\
\hline & & $\begin{array}{l}\text { Initial } \\
\text { Crack }\end{array}$ & $\begin{array}{l}\text { Final } \\
\text { Crack }\end{array}$ & $\begin{array}{l}\text { Initial } \\
\text { Crack }\end{array}$ & $\begin{array}{l}\text { Final } \\
\text { Crack }\end{array}$ & $\begin{array}{l}\text { Initial } \\
\text { Crack }\end{array}$ & $\begin{array}{l}\text { Final } \\
\text { Crack }\end{array}$ \\
\hline Normal Concrete & 0 & 12 & 15 & 18 & 21 & 23 & 26 \\
\hline \multirow{5}{*}{$\begin{array}{l}\text { Concrete with inclusion } \\
\text { of Banana stem fiber }\end{array}$} & 0.5 & 65 & 69 & 73 & 79 & 91 & 95 \\
\hline & 1 & 69 & 71 & 83 & 80 & 110 & 115 \\
\hline & 1.5 & 73 & 77 & 86 & 89 & 121 & 127 \\
\hline & 2 & 78 & 81 & 89 & 93 & 123 & 128 \\
\hline & 2.5 & 82 & 84 & 90 & 95 & 124 & 130 \\
\hline \multirow{5}{*}{$\begin{array}{c}\text { Concrete with inclusion } \\
\text { of Steel fiber }\end{array}$} & 0.5 & 71 & 76 & 80 & 84 & 115 & 118 \\
\hline & 1 & 75 & 79 & 96 & 101 & 146 & 153 \\
\hline & 1.5 & 81 & 85 & 103 & 110 & 155 & 160 \\
\hline & 2 & 83 & 89 & 108 & 113 & 158 & 162 \\
\hline & 2.5 & 83 & 90 & 111 & 117 & 161 & 165 \\
\hline
\end{tabular}

\section{Bond Strength Test}

This investigation involved pullout test on both plain and deformed steel bars and comparison of bond strength with conventional concrete, banana stem fiber concrete, and steel fiber concrete. For bond strength test, specimens of size $200 \mathrm{~mm}$ height and $100 \mathrm{~mm}$ diameter were used and specimens were incorporated with both deformed bars (RTS) and plain bars (MS) of 8, 10, 12 and 16mm ( Table-5). 
RASĀYAN J. Chem.

Vol. 11 | No. 2 |640 - 646 | April - June | 2018

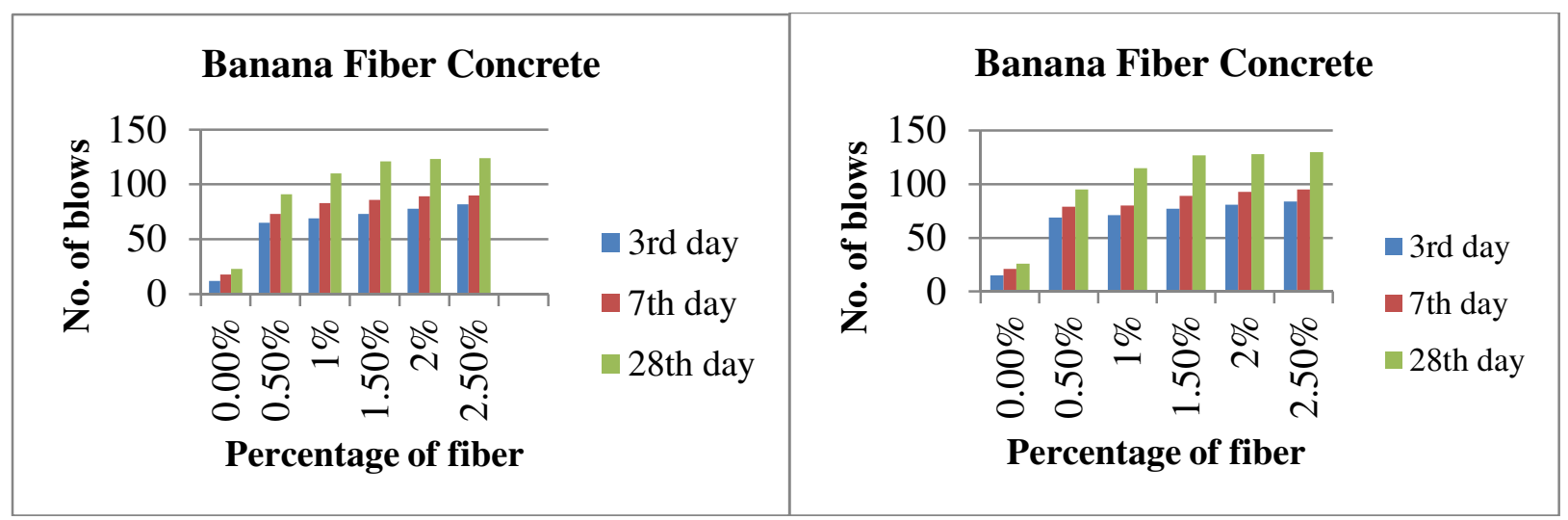

Fig.-4: Comparison of the initial \& final crack of banana stems fiber concrete

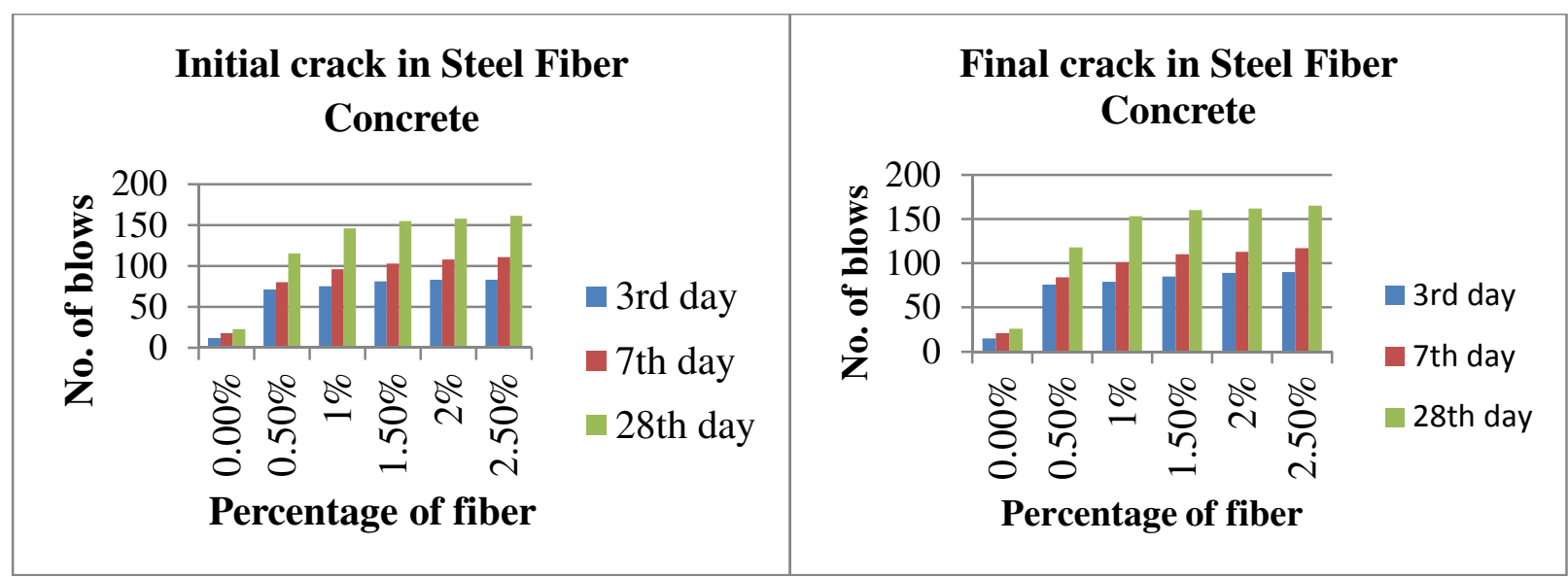

Fig.-5: Comparison of the initial \& final crack of SFRC

Table-5: Bond strength of Conventional Concrete with RTS bars and MS bars

\begin{tabular}{|c|c|c|c|c|c|c|c|c|c|c|c|c|c|}
\hline \multirow{3}{*}{$\begin{array}{l}\text { Diameter } \\
\text { of RTS } \\
\text { bars (mm) }\end{array}$} & \multicolumn{6}{|c|}{ Conventional concrete (CC) } & \multirow{3}{*}{$\begin{array}{c}\text { Diameter } \\
\text { of MS bars } \\
(\mathrm{mm})\end{array}$} & \multicolumn{6}{|c|}{ Conventional concrete (CC) } \\
\hline & \multicolumn{3}{|c|}{$\begin{array}{c}\text { Bond } \\
\text { strength }\left(\mathrm{N} / \mathrm{mm}^{2}\right)\end{array}$} & \multicolumn{3}{|c|}{ Slip (mm) } & & \multicolumn{3}{|c|}{$\begin{array}{c}\text { Bond } \\
\text { strength }\left(\mathrm{N} / \mathrm{mm}^{2}\right)\end{array}$} & \multicolumn{3}{|c|}{ Slip (mm) } \\
\hline & $\begin{array}{l}3^{\text {rd }} \\
\text { day }\end{array}$ & $\begin{array}{l}7^{\text {th }} \\
\text { day }\end{array}$ & $\begin{array}{l}28^{\text {th }} \\
\text { day }\end{array}$ & $\begin{array}{l}3^{\text {rd }} \\
\text { day }\end{array}$ & $\begin{array}{l}7^{\text {th }} \\
\text { day }\end{array}$ & $\begin{array}{l}28^{\text {th }} \\
\text { day }\end{array}$ & & $\begin{array}{l}3^{\text {rd }} \\
\text { day }\end{array}$ & $\begin{array}{c}7^{\text {th }} \\
\text { day }\end{array}$ & $\begin{array}{l}28^{\text {th }} \\
\text { day }\end{array}$ & $\begin{array}{c}3^{\text {rd }} \\
\text { day }\end{array}$ & $\begin{array}{l}7^{\text {th }} \\
\text { day }\end{array}$ & $\begin{array}{l}28^{\text {th }} \\
\text { day }\end{array}$ \\
\hline 8 & 3.86 & 6.5 & 10.6 & 12 & 8.2 & 4 & 8 & 2.75 & 5.61 & 9.03 & 6.14 & 4.34 & 2.10 \\
\hline 10 & 3.02 & 5.31 & 8.28 & 10.4 & 7.2 & 3.8 & 10 & 2.66 & 4.39 & 7.68 & 4.45 & 3.73 & 1.72 \\
\hline 12 & 2.41 & 4.06 & 6.47 & 8.76 & 5 & 1.7 & 12 & 2.01 & 2.29 & 5.62 & 3.84 & 3.44 & 1.48 \\
\hline 16 & 1.67 & 2.63 & 5.01 & 6.26 & 4.7 & 3.4 & 16 & 1.37 & 2.16 & 4.06 & 3.7 & 3.19 & 1.18 \\
\hline
\end{tabular}

Table-6: Bond strength of RTS bars with $1 \%$ of Banana Stem Fiber and SFC

\begin{tabular}{|c|c|c|c|c|c|c|c|c|c|c|c|c|}
\hline \multirow{3}{*}{$\begin{array}{c}\text { The } \\
\text { diameter of } \\
\text { RTS bars } \\
(\mathrm{mm})\end{array}$} & \multicolumn{6}{|c|}{ Banana stem fiber concrete (BFC- $1 \%$ ) } & \multicolumn{6}{|c|}{ Steel fiber concrete (SFC-1\%) } \\
\hline & \multicolumn{3}{|c|}{ Bond strength $\left(\mathrm{N} / \mathrm{mm}^{2}\right)$} & \multicolumn{3}{|c|}{ Slip (mm) } & \multicolumn{3}{|c|}{ Bond strength $\left(\mathrm{N} / \mathrm{mm}^{2}\right)$} & \multicolumn{3}{|c|}{ Slip (mm) } \\
\hline & $\begin{array}{l}3^{\text {rd }} \\
\text { day }\end{array}$ & $\begin{array}{c}7^{\text {th }} \\
\text { day }\end{array}$ & $\begin{array}{l}28^{\text {th }} \\
\text { day }\end{array}$ & $\begin{array}{c}3^{\text {rd }} \\
\text { day }\end{array}$ & $\begin{array}{c}7^{\text {th }} \\
\text { day }\end{array}$ & $\begin{array}{l}28^{\text {th }} \\
\text { day }\end{array}$ & $\begin{array}{c}3^{\text {rd }} \\
\text { day } \\
\end{array}$ & $\begin{array}{c}7^{\text {th }} \\
\text { day }\end{array}$ & $\begin{array}{l}28^{\text {th }} \\
\text { day }\end{array}$ & $\begin{array}{c}3^{\text {rd }} \\
\text { day }\end{array}$ & $\begin{array}{l}7^{\text {th }} \\
\text { day }\end{array}$ & $\begin{array}{l}28^{\text {th }} \\
\text { day }\end{array}$ \\
\hline 8 & 3.7 & 6.37 & 9.79 & 11.8 & 8.7 & 5.21 & 4.33 & 6.80 & 11.8 & 12 & 8.7 & 4.7 \\
\hline 10 & 2.85 & 5.18 & 7.29 & 9.86 & 7.56 & 4.82 & 3.43 & 5.61 & 9.08 & 10.4 & 8 & 4.4 \\
\hline 12 & 2.29 & 4.03 & 5.78 & 8.6 & 6.6 & 3.2 & 2.54 & 4.89 & 7.3 & 8.85 & 6.4 & 2.5 \\
\hline 16 & 1.58 & 2.58 & 4.03 & 6.5 & 5 & 4.1 & 1.86 & 2.73 & 5.56 & 6.29 & 5.1 & 3.8 \\
\hline
\end{tabular}


RASĀYAN J. Chem.

Vol. 11 | No. 2 |640 - 646 | April - June | 2018

Table-7: Bond test result of MS bars with $1 \%$ of Banana Stem Fiber and SFC

\begin{tabular}{|c|c|c|c|c|c|c|c|c|c|c|c|c|}
\hline \multirow{3}{*}{$\begin{array}{l}\text { The } \\
\text { diameter of } \\
\text { MS bars } \\
(\mathrm{mm})\end{array}$} & \multicolumn{6}{|c|}{ Banana stem fiber concrete (BFC- $1 \%$ ) } & \multicolumn{6}{|c|}{ Steel fiber concrete (SFC- $1 \%$ ) } \\
\hline & \multicolumn{3}{|c|}{ Bond strength $\left(\mathrm{N} / \mathrm{mm}^{2}\right)$} & \multicolumn{3}{|c|}{ Slip (mm) } & \multicolumn{3}{|c|}{ Bond strength $\left(\mathrm{N} / \mathrm{mm}^{2}\right)$} & \multicolumn{3}{|c|}{ Slip (mm) } \\
\hline & $\begin{array}{l}3^{\text {rd }} \\
\text { day }\end{array}$ & $\begin{array}{l}7^{\text {th }} \\
\text { day }\end{array}$ & $\begin{array}{l}28^{\text {th }} \\
\text { day }\end{array}$ & $\begin{array}{c}3^{\text {rd }} \\
\text { day }\end{array}$ & $\begin{array}{l}7^{\text {th }} \\
\text { day }\end{array}$ & $\begin{array}{l}28^{\text {th }} \\
\text { day }\end{array}$ & $\begin{array}{c}3^{\text {rd }} \\
\text { day }\end{array}$ & $\begin{array}{c}7^{\text {th }} \\
\text { day }\end{array}$ & $\begin{array}{l}28^{\text {th }} \\
\text { day }\end{array}$ & $\begin{array}{c}3^{\text {rd }} \\
\text { day }\end{array}$ & $\begin{array}{l}7^{\text {th }} \\
\text { day }\end{array}$ & $\begin{array}{l}28^{\text {th }} \\
\text { day }\end{array}$ \\
\hline 8 & 3.06 & 6.01 & 10.08 & 6.14 & 4.1 & 2.56 & 2.91 & 5.28 & 7.38 & 5.82 & 3.82 & 2.81 \\
\hline 10 & 2.84 & 4.48 & 8.59 & 4.49 & 3.4 & 2.18 & 2.65 & 4.27 & 6.37 & 4.29 & 3.13 & 2.13 \\
\hline 12 & 2.29 & 2.69 & 6.39 & 3.86 & 3.42 & 1.97 & 1.88 & 2.19 & 4.81 & 3.57 & 2.69 & 1.68 \\
\hline 16 & 1.68 & 2.38 & 4.73 & 3.78 & 3.19 & 1.68 & 1.42 & 2.01 & 3.49 & 3.18 & 2.47 & 1.32 \\
\hline
\end{tabular}

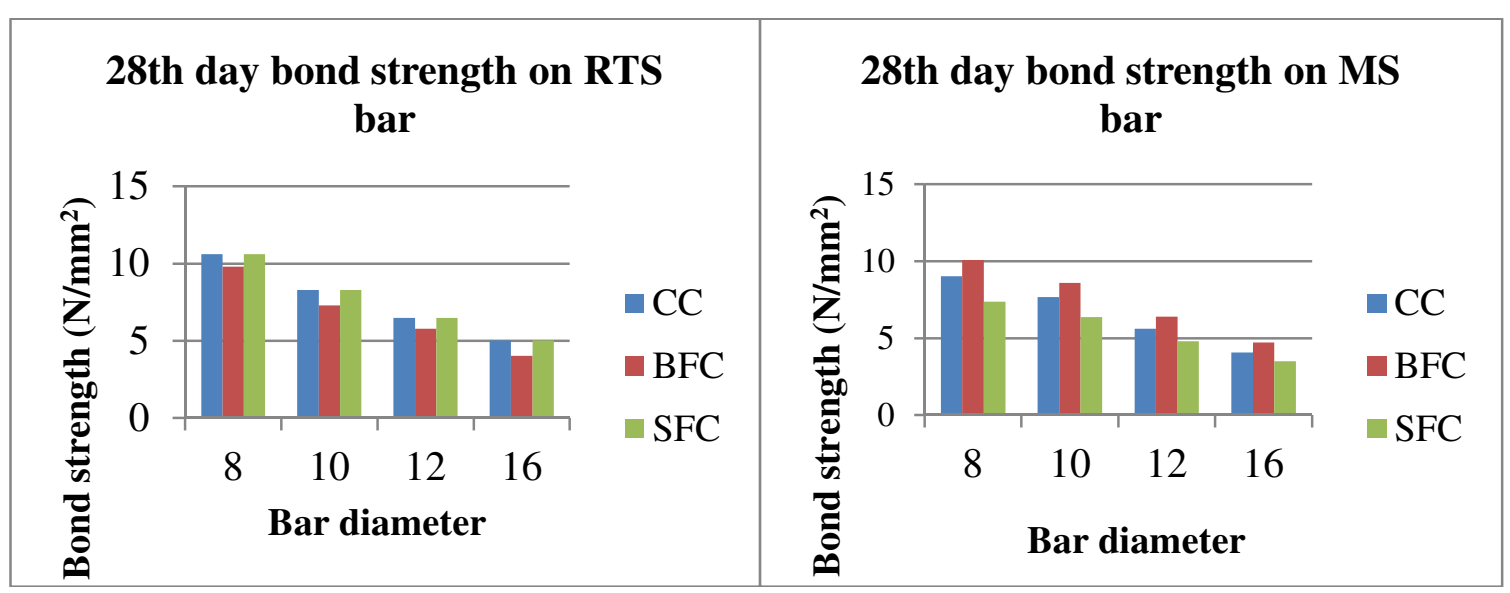

Fig.-6: Comparison of bond strength of RTS \& MS bars with CC and fiber concrete at $28^{\text {th }}$ day

\section{CONCLUSION}

- From the test result it can be seen that, with the presence of both banana stem and steel fiber in concrete, the compressive, tensile and flexural strength found to be optimum at $1 \%$ volume fraction of fiber.

- It was observed that steel fiber reinforced concrete is having better compressive, tensile, flexural, and impact strength as compared to banana stem fiber.

- It can be seen that beyond $1 \%$ volume fraction of fiber content in concrete there is a gradual decrease in compressive, tensile and flexural strength.

- The impact strength of both type concrete observed to be increased as the percentage of banana stem fibers and steel fibers are increased and also steel reinforced fiber concrete having more impact strength than banana stem fiber concrete.

- It was observed that inclusion of steel fiber in concrete changes the brittle form of concrete to ductile and improved impact resistance.

- Fibered concrete specimens with deformed bars as well as plain bars are comparable with conventional concrete. Conventional concrete with steel fiber having more bond strength compared to banana stem fiber concrete.

- It was observed that both normal and steel reinforced concrete with deformed bars showing improved bond strength at the age of 28 days.

- . Based on this result, we can conclude that the addition of both banana stem fiber and steel fiber emphasizes the basic physical properties of concrete. But steel fibers are superior as compared to banana stem fiber.

\section{REFERENCES}

1. S. Prakash Chandar, K. Gunasekaran, N. Sai Sandeep, S. Manikandaprabhu, Rasayan Journal of Chemistry, 10(2), 528(2017), DOI: 10.7324/RJC.2017.1021636 
2. N. Ganapathy Ramasamy, R. Dhanya, et al., Rasayan Journal of Chemistry, 10(2), 577(2017), DOI: $10.7324 /$ RJC.2017.1021671

3. R. Ramasubramani, K.S. Sathyanarayanan, et al., Rasayan Journal of Chemistry, 9(4), 706(2016)

4. K. Divya Krishnan, P.T. Ravichandran, V.K. Gandhimathi, Rasayan journal of chemistry, 10(2), 600(2017), DOI: 10.7324/RJC.2017.1021677

5. A. M. Shende, A.M. Pande and Gulfam Pathan, International Refereed Journal of Engineering and Science, 1(1), 48(2012)

6. Job Thomas and Ananth Ramaswamy, Journal of Materials in Civil Engineering, 19(5), 385(2007), DOI: 10.0161/(ASCE)0899-1561(2007)

7. K.L. Pickering, M.G Aruan Efendy, T.M Le, Applied Science and Manufacturing, 83, 98(2016), DOI: 10.1016/j.compositesa

8. Ravi Bhatnagar, Gourav Gupta, Sachin Yadav, International Journal of Scientific and Engineering Research, 6(5), 49(2015)

9. Layth Mohammed, M. N. M. Ansari, Gracepua, Mohammed Jawaid, M. Saiful Islam, International Journal of Polymer Science, 2015, (2015), DOI: 10.1155/2015/243947

10. Samrat Mukhopadhyay and Bishwajit Bhattacharjee, Journal of Industrial Textiles, 45(5), 957(2016), DOI: 10.1177/1528083714545394

11. Abdul Ghaffar, Amit S. Chavhan, R.S. Tatwawadi, International Journal of Engineering Trends and Technology, 9(15), 797(2014), DOI: 10.14445/22315381/IJETT

[RJC-3011/2018] 